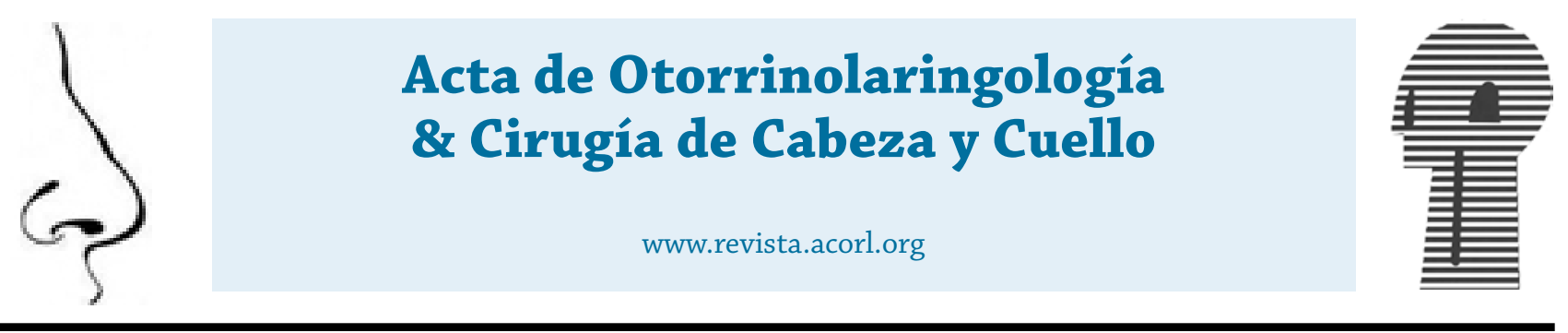

\title{
Evaluación de los desenlaces posoperatorios de los pacientes sometidos a septoplastia y turbinoplastia entre los años 2016 a 2018 de la Fundación Santa Fe de Bogotá
}

\section{Evaluation of postoperative outcomes of septoplasty and septoturbinoplasty patients of Fundación Santa Fe de Bogota between the years 2016 to 2018}

\author{
Aldana-Gallego $G^{*}$, Peñaranda-Sanjuan $A^{* *}$, Moreno SM***, Pérez-García IC****
}
* MD; otorrinolaringóloga. Fundación Santa Fe de Bogotá. Universidad de los Andes. Dirección: Calle 119 \# 7 - 90. Teléfono: 60303030. E-mail: gilmaldanagallego@yahoo.com. ORCID: https://orcid.org/0000-0002-7248-0280
** MD, Msc; otorrinolaringólogo, otólogo, epidemiólogo clínico. Fundación Santa Fe de Bogotá. Universidad de los Andes. Dirección: Calle 119 \# 7-90. Teléfono: 60303030. ORCID: https://orcid.org/0000-0003-1598-8472
*** Msc; estadístico, epidemiólogo clínico. Fundación Santa Fe de Bogotá. Universidad de los Andes. Dirección: Calle 119 \# 7-90. Teléfono: 60303030. ORCID: https://orcid.org/0000-0002-3043-0963
**** MD; otorrinolaringóloga. Fundación Santa Fe de Bogotá. Universidad de los Andes. Dirección: Calle 119 \# 7-90. Teléfono: 60303030. E-mail:irecami@gmail.com. ORCID: https://orcid.org/0000-0002-7248-0280

\footnotetext{
Forma de citar: Aldana-Gallego G, Peñaranda-Sanjuan A, Moreno SM, Pérez-García IC. Evaluación de los desenlaces posoperatorios de los pacientes sometidos a septoplastia y turbinoplastia entre los años 2016 a 2018 de la Fundación Santa Fe de Bogotá. Acta otorrinolaringol. cir. cabeza cuello. 48(3): 226-231 Doi:10.37076/acorl.v48i3.503
}

\section{INFORMACIÓN DEL ARTÍCULO}

Historia del artículo:

Recibido: 4 de mayo de 2020

Evaluado: 19 de agosto de 2020

Aceptado: 28 de agosto de 2020

\section{Palabras clave (DeCS):}

Procedimientos quirúrgicos nasales hematoma

\section{Correspondencia:}

Nombre Gilma Aldana Gallego

E-mail: gilmaldanagallego@yahoo.com

Dirección:Calle 119 \# 7-90.

Teléfono celular:3012040007

\section{RESUMEN}

Introducción: La tasa de infección después de la cirugía nasal electiva es muy baja, lo que hace que la profilaxis antibiótica de rutina sea redundante. En Colombia no disponemos de información acerca de la tasa de infección de septoplastia; por esta razón, en este artículo se busca describir la tasa de infección posquirúrgica en los pacientes llevados a septoplastia o septoplastia y turbinoplastia de la Fundación Santa Fe de Bogotá, entre los años 2016-2018. Metodología: se realizó un estudio observacional, descriptivo y retrospectivo mediante la revisión de la base de datos del servicio. Se calculó la proporción de infección para todos los individuos participan- 
tes del estudio de manera general y estratificada por el uso de antibiótico profiláctico y posoperatorio, y se describió la frecuencia de las complicaciones. Resultados: encontramos 255 pacientes en la base de datos de la sección de otorrinolaringología, de los cuales 206 cumplieron con los criterios de inclusión. El 23,3 \% de los pacientes recibió un antibiótico profiláctico y el $24,76 \%$ recibió un antibiótico postoperatorio. La tasa de infección posoperatoria fue de 2,91\% (intervalo de confianza [IC] del 95 $\%$ : 1,07-6,23). El 96,6\% de los pacientes no presentaron complicaciones. Conclusiones: nuestros hallazgos se correlacionan con la literatura global.

\section{ABSTRACT}

Key words (MeSH):

Nasal surgical procedures henorlhage hematoma
Introduction: infection rate after elective nasal surgery is very low which makes routine antibiotic prophylaxis redundant. In Colombia we do not have information about postoperative infection in septoplasty. A study was designed to determine the postoperative infection rate in patients undergoing septoplasty or septoplasty and turbinoplasty at Fundación Santa Fe de Bogotá between 2016 to 2018. Methods: an observational, descriptive and retrospective study was conducted. The database of the Otolaryngology Section was reviewed. The proportion of infection for all individuals participating in the study was calculated in a general and stratified manner by use of prophylactic and postoperative antibiotic and the frequency of complications was described. Results: we found 255 patients in the patient database of which 206 patients met the inclusion criteria. $23.3 \%$ of patients received prophylactic antibiotic and $24.76 \%$ received postoperative antibiotic. The percentage of postoperative infection was $2.91 \%$ (95\% CI: 1.07-6.23). $96.6 \%$ of the patients did not present any complications. Conclusion: septoplasty has a low risk of infection which was in accordance to the findings found in the present study.

\section{Introducción}

La revisión de Georgio y colaboradores sugiere que la tasa de infección después de la cirugía nasal electiva es muy baja (1), lo que hace que la profilaxis antibiótica de rutina sea redundante. Los antibióticos sistémicos preventivos están indicados en las rinoplastias de revisión complicadas, la colocación prolongada de taponamientos nasales y en pacientes que son susceptibles a las infecciones $(1,2)$. En un estudio que realizaron Itsmi y colaboradores (3) usando una sutura transeptal y/o láminas de silicona, se encontró que la colonización por Staphylococcus aureus en la cavidad nasal se pudo prevenir con estas técnicas, en vez de aplicar un tratamiento antibiótico profiláctico en pacientes sanos.

En el contexto de Colombia, no disponemos de información acerca de la infección en cirugías como la septoplastia con o sin uso de antibiótico perioperatorio. En 2015 se implementó la guía de manejo de septoplastia y septoturbinoplastia en la Fundación Santa Fe, en la cual se siguieron las recomendaciones de la Academia Americana de Otorrinolaringología sobre la no utilización de antibiótico perioperatorio de rutina en pacientes sin factores de riesgo.

Ante ese vacío de conocimiento decidimos realizar este estudio, el cual busca responder las siguientes preguntas: ¿cuál es la tasa de infección posquirúrgica en los pacientes llevados a septoplastia o septoplastia y turbinoplastia de la Fundación Santa Fe de Bogotá desde el año 2016 hasta el año 2018? ¿Cuáles son las características de los pacientes con dichas infecciones?

\section{Materiales y métodos}

Se realizó un estudio observacional, descriptivo, de tipo retrospectivo de los pacientes sometidos a septoplastia o septoplastia y turbinoplastia del Hospital Universitario Fundación Santa Fe de Bogotá (FSFB) desde el año 2016 hasta el año 2018, mediante la revisión de la base de datos de la sección de otorrinolaringología.

Se incluyeron todos los pacientes entre 13 y 82 años sometidos a septoplastia o septoplastia y turbinoplastia por parte de los cirujanos de la sección de otorrinolaringología del Hospital Universitario Fundación Santa Fe de Bogotá desde el año 2016 hasta el año 2018, quienes cumplieron con los criterios de inclusión (Tabla 1).

Se registraron a los individuos de forma consecutiva desde el inicio hasta el final del período mencionado, y se realizó la revisión de las historias clínicas de estos pacientes en el sistema de la FSFB en busca de información acerca de los resultados investigados. Con el propósito de mitigar la posible pérdida de información, se realizó una revisión por pares de la información consignada en la malla de recolección por parte de las investigadoras principales. En total, ingresaron al estudio 206 pacientes.

\section{Aspectos éticos}

Este estudio se realizó conforme con la Declaración de Helsinki y sus principios éticos para las investigaciones médicas en seres humanos, y fue desarrollado por profesionales 
Tabla 1. Criterios de inclusión y de exclusión

Criterios de inclusión

- Pacientes llevados a septoplastia en la Fundación Santa Fe de Bogotá.

- Pacientes llevados a septoplastia y turbinoplastia en la Fundación Santa Fe de Bogotá.

- Pacientes llevados a cirugía entre enero de 2016 y diciembre de 2018.

\begin{tabular}{|l|}
\hline \multicolumn{1}{|c|}{ Criterios de exclusión } \\
\hline - Mujeres embarazadas. \\
\hline - Síndromes craneofaciales. \\
\hline - Pacientes con perforación septal, ya sea primaria o secundaria \\
a algún procedimiento quirúrgico. \\
\hline - Radiación en cabeza y el cuello. \\
\hline - Rinosinusitis aguda o crónica. \\
\hline - Tumores benignos o malignos nasosinusales. \\
\hline - Pacientes llevados a septoplastia , en el mismo tiempo \\
quirúrgico, a otros procedimientos diferentes a turbinoplastia. \\
\hline
\end{tabular}

expertos en las áreas de cirugía, otorrinolaringología y estadística con la aprobación del Comité de ética médica de la Fundación Santa Fe y fue aprobado con el código CCEI11474-2019.

\section{Procedimiento quirúrgico}

El procedimiento se realiza usando fotóforo, con el paciente bajo anestesia general; se protegen los ojos y se aplica una solución antiséptica tipo clorhexidina en la parte externa de la nariz. Se utiliza un anestésico local (lidocaína con 1:100 000) mínimo 5 minutos antes de la incisión, la cual es hemitransfixiante en la fosa nasal a elegir en el margen caudal del cartílago septal, usando un bisturí número 15. A continuación, se realiza una elevación cuidadosa del colgajo mucopericóndrico en dirección posterior con el elevador de Cottle o Freer. Se preservan al menos $1,5 \mathrm{~cm}$ de los bordes cartilaginosos caudal y dorsal. Posteriormente, se realiza condrotomía con hoja de bisturí número 15 o Freer agudo de las deformidades identificadas antes de la cirugía. Finalmente, se reafrontan los colgajos mucopericóndricos y se cierra la incisión con sutura absorbible. Según el cirujano, se posicionan láminas de Doyle, Merocel, Surgicel o mechas nasales o solo suturas transfixiantes.

\section{Análisis estadístico}

Se calcularon frecuencias (absolutas y relativas) y porcentajes para las variables cualitativas, y medidas de tendencia central (media y mediana) y dispersión (desviación estándar) junto con los valores máximos y mínimos para las variables cuantitativas. Para ambos tipos de variables, se realizaron algunos gráficos descriptivos.

Se calculó la proporción de infección para todos los individuos participantes del estudio de manera general y estratificada por el uso de antibiótico profiláctico y posoperatorio.
Adicionalmente, se describió la frecuencia de complicaciones: hematomas septales, abscesos septales y otras complicaciones posquirúrgicas, de manera general y estratificada. El software de análisis utilizado fue Stata 13 SE.

\section{Resultados}

En total, se encontraron 255 pacientes en la base de datos de la sección de otorrinolaringología de la Fundación Santa Fe de Bogotá, a los que se les realizó septoplastia o septoturbinoplastia en la misma institución, entre los años 2016 y 2018. De estos, 49 pacientes fueron sometidos a cirugías adicionales a la septoplastia como rinoplastia, reducción de fracturas nasales, entre otras, por lo que fueron excluidos del estudio. En este sentido, quedaron un total de 206 pacientes, quienes cumplieron con los criterios de inclusión.

Del total de la población de estudio, 139 fueron hombres $(67,48 \%)$. La media de edad fue de 31,3 años (desviación estándar $[\mathrm{DE}]=15,37)$, edad mínima de 7 y máxima de 71 años; el 71,8 \% de los pacientes pertenecen a medicina prepagada. Las características demográficas de los pacientes se pueden apreciar en la Tabla 2.

Tabla 2. Características demográficas

\begin{tabular}{|l|c|c|}
\hline \multirow{2}{*}{ Variables } & \multicolumn{2}{c|}{$\mathrm{n}=206$} \\
\cline { 2 - 3 } & $\mathrm{n}$ & $\%$ \\
\hline Sexo & 139 & 67,48 \\
\hline Masculino & $35,26(15,37)$ & $33,5(7-71)$ \\
\hline Edad en años(a) & 1 & 0,49 \\
\hline Régimen de salud & 148 & 71,84 \\
\hline Contributivo & 34 & 16,50 \\
\hline Prepagada & 1 & 0,49 \\
\hline Plan complementario & 6 & 2,91 \\
\hline SOAT & 16 & 7,77 \\
\hline Seguro internacional & 6 & 2,91 \\
\hline Pago directo & 2 & 0,97 \\
\hline Antecedentes clínicos relevantes & 36,41 \\
\hline Hipertensión arterial & 75 & \\
\hline Apnea del sueño &
\end{tabular}

(a) Valores continuos reportados en medias (DE) y Medianas (mín. - máx.)

Respecto a los antecedentes médicos de los pacientes, $123(59,7 \%)$ no presentaban ningún antecedente y $75(36,4$ $\%)$ tenían otros antecedentes como rinitis alérgica, dislipidemia, entre otros. Ningún paciente llevado a cirugía consumía aspirina, tenía alguna discrasia sanguínea, tenía anticoagulación o sufría de una enfermedad protrombótica (Tabla 2).

En cuanto al taponamiento nasal realizado en cirugía después de la septoplastia y antes del despertar, el $73 \%$ de la 
población del estudio tuvo algún tipo de taponamiento nasal y el $19 \%$ no tuvo ningún tipo de taponamiento. En estos pacientes se realizó sutura septal transfixiante con material absorbible. En el $8 \%$ de los pacientes no se mencionó en la historia clínica si se puso o no taponamiento nasal, por lo que se desconocen los datos (Figura 1).

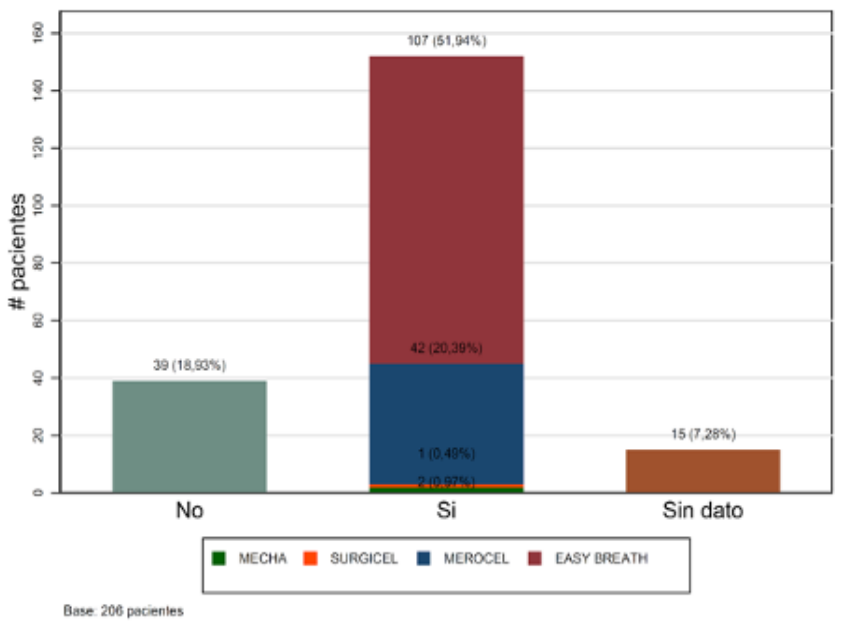

Figura 1. Número de pacientes a los que se les realizó taponamiento nasal en cirugía y tipo de taponamiento nasal.

Tabla 3. Uso de antibiótico perioperatorio, sangrado posoperatorio y complicaciones

\begin{tabular}{|l|c|c|}
\hline \multirow{2}{*}{$\begin{array}{c}\text { Condiciones del paciente en } \\
\text { el posoperatorio }\end{array}$} & \multicolumn{2}{|c|}{$\mathrm{n}=206$} \\
\cline { 2 - 3 } & $\mathrm{n}$ & $\%$ \\
\hline Presencia de & \multicolumn{2}{|}{} \\
\hline Antibiótico profiláctico & 48 & 23,30 \\
\hline Antibiótico posoperatorio & 51 & 24,76 \\
\hline Sangrado posoperatorio & 1 & 0,49 \\
\hline Complicaciones posoperatorias \\
\hline Absceso septal & 2 & 0,97 \\
\hline Epistaxis leve & 1 & 0,49 \\
\hline Ninguna & 199 & 96,60 \\
\hline Sin dato & 4 & 1,94 \\
\hline
\end{tabular}

En cuanto al uso de antibiótico perioperatorio, el 23,3\% de los pacientes recibió antibiótico profiláctico (antes del inicio de la cirugía, usualmente se administran cefalosporinas si no hay contraindicación) y el $24,76 \%$ recibió antibiótico posoperatorio (usualmente un betalactámico).

El porcentaje de infección posoperatoria fue de 2,91\% (intervalo de confianza [IC] del $95 \%: 1,07-6,23$ ). Dos pacientes $(\mathrm{n}=2)$ presentaron abscesos septales que requirieron drenaje y taponamiento nasal y no recibieron ningún antibiótico perioperatorio ni posoperatorio; un paciente de 33 años con antecedente de apnea del sueño egresó de cirugía con láminas de Doyle o Easy Breath; otro paciente de 17 años sin ningún antecedente importante no tuvo ningún tipo de taponamiento. Fueron procedimientos realizados por dos ci- rujanos diferentes.

El 96,6\% de los pacientes no presentaron complicaciones. La complicación más frecuente fue el absceso septal presente en los dos pacientes mencionados previamente. Un paciente presentó epistaxis leve posterior a la cirugía. Este paciente de 24 años no tenía ningún antecedente importante y no tuvo taponamiento nasal puesto en cirugía (Tabla 4).

Tabla 4. Presencia de infección posoperatoria estratificada por la presencia de infección en el posoperatorio [Nota: es necesario añadir el llamado de esta tabla dentro del texto]

\begin{tabular}{|r|c|c|c|c|}
\hline \multirow{2}{*}{$\begin{array}{c}\text { Uso de } \\
\text { antibiótico }\end{array}$} & \multicolumn{3}{|c|}{ Presencia de infección en el posoperatorio } \\
\cline { 2 - 5 } & $\mathrm{n}(\mathrm{a})$ & $\%(\mathrm{a})$ & $\mathrm{n}(\mathrm{a})$ & $\%(\mathrm{a})$ \\
\hline Antibiótico profiláctico & \multicolumn{3}{c|}{ Sí = 2 } \\
\hline No & 156 & 76,47 & 2 & 100,00 \\
\hline Sí & 48 & 23,53 & 0 & 0,00 \\
\hline Antibiótico en el posoperatorio & \\
\hline No & 141 & 69,12 & 2 & 100,00 \\
\hline Sí & 51 & 25,00 & 0 & 0,00 \\
\hline
\end{tabular}

(a) Porcentajes calculados con base en el número de sujetos con y sin infección.

\section{Discusión}

La obstrucción nasal es el síntoma más común en la práctica rinológica, y el septo desviado es la causa más común de obstrucción nasal. Se ha estimado que al menos un tercio de la población tiene algo de obstrucción nasal, y que al menos un cuarto de estos pacientes requiere algún tratamiento quirúrgico (2).

Se ha estimado un costo anual de 5 billones de dólares para aliviar la obstrucción nasal, y aproximadamente $60 \mathrm{mi}-$ llones de dólares en procedimientos quirúrgicos (4). Además de obstrucción nasal, un septo significativamente desviado se ha correlacionado con diferentes patologías como sinusitis, cefaleas (atribuibles a puntos de contacto con estructuras de la pared lateral nasal), incluso pérdida de la calidad de vida de quien la padece. Se ha evidenciado que los pacientes con deflexiones septales tienen más riesgo de epistaxis, que son un factor agravante de la apnea del sueño y pueden producir una mayor obstrucción nasal en los pacientes con rinitis alérgica (5). Cuando los pacientes con desviación septal se someten a septoplastia, tienen una mejoría significativa en su sintomatología y en su calidad de vida $(6,7)$.

Por esta razón, la septoplastia es la cirugía que se realiza con más frecuencia en la práctica de la otorrinolaringología, por lo que es de vital importancia comprender las conductas perioperatorias que realizamos como cirujanos y su impacto en nuestros pacientes.

En este estudio encontramos que la mayoría de los pacientes sometidos a septoplastia en nuestra institución son hombres. No está descrito en la literatura que las desviaciones septales sean más frecuentes en hombres que en mujeres, por lo cual llama la atención este hallazgo, y sería interesante iden- 
tificar la causa. También se encontró que, en la FSFB, este es un procedimiento que se realiza más en pacientes en la cuarta década de la vida, aunque con un rango muy grande, ya que es una cirugía que puede requerirse en niños si las desviaciones septales son muy obstructivas, o en adultos especialmente en el contexto del tratamiento de la apnea del sueño.

Debido a las características de los convenios de nuestra institución, la mayoría de los pacientes (71\%) sometidos a septoplastia pertenecen a instituciones de medicina prepagada. Esto no es lo usual en el contexto colombiano, en donde se estima que la población afiliada a medicina prepagada es de 1,5 millones de personas únicamente (8). Este hallazgo hace que nuestros resultados sean menos universales y extrapolables a otras instituciones prestadoras de salud.

La septoplastia es una cirugía que, en nuestra institución, en general se realiza en pacientes sin antecedentes médicos importantes. Nos pareció interesante analizar si nuestros pacientes recibían algún medicamento que produjera antiagregación o anticoagulación y su relación con el sangrado posoperatorio, pero ninguno de los pacientes reclutados para este estudio estaba siendo tratado con este tipo de medicamentos. También es posible que los pacientes que están bajo estos tratamientos, no se lleven con frecuencia a esta cirugía debido al riesgo que implican.

La mayoría de procedimientos quirúrgicos fueron realizados por dos cirujanos, quienes son dos de las personas con mayor antigüedad en la institución. Esto explica porque manejan un mayor volumen de pacientes. Posteriormente y durante los años del análisis, ingresaron cuatro otorrinolaringólogos a la institución, que para el momento del estudio manejaron un menor volumen de pacientes.

En 2015, en nuestra institución, se adoptó una guía de manejo de septoplastia con y sin turbinoplastia, en la que se estandarizó evitar el uso de taponamiento nasal con Merocel, Surgicel o mechas, y en su lugar se recomienda la utilización de láminas tipo Doyle o Easy Breath, así como no usar antibióticos perioperatorios de rutina en nuestros pacientes. Los pacientes reclutados para este estudio fueron intervenidos después de la adopción de esta guía, algunos otorrinos no adoptaron inicialmente la guía por lo cual en el $21 \%$ de los pacientes se utilizó taponamiento nasal con los materiales mencionados, y en el $52 \%$ el uso de láminas de Doyle. En nuestra institución casi ningún paciente requiera de rutina taponamiento con Merocel o mechas, por lo cual este estudio puede contribuir a demostrar que el uso de estas láminas de silicona no aumenta el riesgo de sangrado o infección.

La tasa de sangrado posoperatorio en este estudio fue de $0,48 \%$ ( 1 paciente), una tasa inferior a lo descrito por la literatura. Las láminas de Doyle, cuando no permanecen un período de tiempo prolongado, no requieren de antibioticoterapia. A pesar de que administrar antibiótico posoperatorio es una práctica clínica frecuente durante el tiempo de permanencia de las láminas, en el estudio de Ismi y colaboradores (3) se encontró que la colonización por Staphylococcus spp. meticilino sensible en la cavidad nasal puede prevenirse con el uso de estas láminas nasales, (diferencia estadísticamente significativa con el taponamiento nasal) y, por ende, se previene el uso de antibiótico perioperatorio.

Respecto al uso de antibiótico perioperatorio, que es uno de los puntos con mayor debate en nuestra institución, encontramos que solo dos pacientes tuvieron infección posoperatoria en forma de absceso septal $(0,97 \%)$. La tasa de infección para septoplastia descrita por Georgiu y colaboradores $(1,2)$ es del $2 \%$, por lo que podemos afirmar que tenemos una baja tasa de infección. Los dos pacientes que tuvieron abscesos septales no recibieron antibiótico perioperatorio; sin embargo, creemos que esta complicación no se debe a la ausencia de este, sino a la probabilidad que tiene la cirugía de tenerla. Estudios han demostrado que la cirugía nasal es limpia-contaminada $(1,5)$ y no necesita antibioticoterapia posoperatoria, dado el bajo riesgo de infección. Adicionalmente, se ha demostrado que los antibióticos sistémicos posoperatorios no disminuyen la morbilidad ni la infección en la septoplastia. La tasa de infección de la cirugía nasal electiva es muy baja, por lo que la profilaxis antibiótica es redundante.

122 pacientes en el estudio (59\%) no recibieron antibiótico perioperatorio; de estos, el $97 \%$ no tuvieron ninguna complicación infecciosa, por lo que podemos concluir que la conducta adoptada en las guías de manejo es adecuada y que podemos continuarla.

A pesar de que las complicaciones después de una septoplastia son raras, los abordajes conservadores con un adecuado diagnóstico preoperatorio minimizan estas complicaciones aún más. La incidencia de complicaciones en la septoplastia es de 3,42\% (9); la tasa de complicaciones en nuestro estudio fue de $1,45 \%$.

Hay algunas limitaciones en nuestra investigación. La primera es que la población que manejamos tiene un mejor y mayor acceso a los servicios de salud, lo cual limita la extrapolación a algunas zonas de nuestro país. La segunda es el tipo de consulta externa manejada por los especialistas de nuestra institución. Algunos especialistas de nuestro grupo realizan consulta externa en sus consultorios con un software de historias clínicas diferente al de la FSFB y realizan la cirugía en la FSFB, por lo que al revisar estas historias clínicas no tenemos información del seguimiento posoperatorio, solo del procedimiento quirúrgico. Estos especialistas reportan sus complicaciones al equipo; sin embargo, al no estar disponibles en el sistema de la FSFB son susceptibles a sesgos. La tercera es el tiempo de análisis que comprende un período previo a la implementación de la guía de manejo y un tiempo después. Sería interesante analizar la información en unos años para evaluar la adherencia a esta guía y evaluar si la tasa de sangrado, la infección y otras complicaciones cambian. La cuarta es el reporte que realizamos; en algunas historias clínicas no estaba consignada toda la información que buscábamos, adicionalmente el cirujano puede no consignar todas las complicaciones o hallazgos al no considerarlos importantes o al encontrarnos mucho más adelante en el seguimiento, como las perforaciones septales. Un paso a seguir es que se estandaricen los datos que deben consignarse en la historia 
clínica y el seguimiento mínimo que debe hacerse a los pacientes después de la cirugía.

\section{Conclusiones}

La septoplastia es uno de los principales procedimientos quirúrgicos adecuados para mejorar la función nasal y la calidad de vida. Según la literatura global, esta cirugía es de bajo riesgo de infección, lo cual se relaciona con los hallazgos en el presente estudio; si el paciente no requiere taponamiento nasal prolongado, no se indica el manejo antibiótico perioperatorio. Igualmente, el sangrado posoperatorio y otras complicaciones tuvieron una tasa baja en nuestra institución.

Dado que nuestra población mayoritariamente pertenece a instituciones de medicina prepagada, nuestros resultados son menos universales y extrapolables a otras instituciones prestadoras de salud; por esta razón, se requieren más estudios prospectivos multicéntricos en otras instituciones de Colombia para la creación e implementación de guías de manejo nacionales.

\section{Declaración de conflicto de intereses}

Los autores no tienen ningún conflicto que declarar. Este artículo se presentó en el XL Congreso de Nacional de otorrinolaringología, entre 11-14 de marzo, en Bucaramanga, Colombia.

\section{Financiación}

Ninguna.

\section{Contribución}

Gilma Aldana Gallego, MD contribuyó con la concepción del artículo, la recolección de datos y el desarrollo de manuscrito; Irene Camila Pérez, MD, con la concepción del artículo, la recolección de datos y el desarrollo del manuscrito; $\mathrm{Au}$ gusto Peñaranda Sanjuan, MD, Msc, con la concepción del artículo y el desarrollo del manuscrito; y Sergio Mauricio Moreno, Msc, con el análisis de datos y el desarrollo del manuscrito.

\section{REFERENCIAS}

1. Georgiou I, Farber N, Mendes D, Winkler E. The role of antibiotics in rhinoplasty and septoplasty: a literature review. Rhinology. 2008;46(4):267-270.

2. Wiesmiller K, Keck T, Rettinger G, Leiacker R, Dzida R, Lindemann J. Nasal air conditioning in patients before and after septoplasty with bilateral turbinoplasty. Laryngoscope. 2006;116(6):890-894. doi:10.1097/01.mlg.0000201995.02171.ea

3. Ismi O, Ozcan C, Vayısoğlu Y, Öztürk C, Tek SA, Görür K. Transseptal suturing technique in septoplasty: impact on bacteremia and nosocomial colonization. Eur Arch Otorhinolaryngol. 2017;274(5):2189-2195. doi:10.1007/ s00405-017-4479-x

4. Rhee JS, Book DT, Burzynski M, Smith TL. Quality of life assessment in nasal airway obstruction. Laryngoscope. 2003;113(7):1118-1122. doi:10.1097/00005537-20030700000004

5. Krouse JH. The unified airway--conceptual framework. Otolaryngol Clin North Am. 2008;41(2):257-v. doi:10.1016/j. otc. 2007.11 .002

6. Moche JA, Palmer O. Surgical management of nasal obstruction. Oral Maxillofac Surg Clin North Am. 2012;24(2):229-viii. doi:10.1016/j.coms.2012.01.013

7. Chandra RK, Patadia MO, Raviv J. Diagnosis of nasal airway obstruction. Otolaryngol Clin North Am. 2009;42(2):207-vii. doi:10.1016/j.otc.2009.01.004

8. Colsanitas, 2019 [Nota: por favor suministrar los datos completos de esta referencia]

9. Dąbrowska-Bień J, Skarżyński PH, Gwizdalska I, Łazęcka $\mathrm{K}$, Skarżyński H. Complications in septoplasty based on a large group of 5639 patients. Eur Arch Otorhinolaryngol. 2018; 275(7): 1789-1794. Published online 2018 May 16. doi: $10.1007 / \mathrm{s} 00405-018-4990-8$ 